\title{
Poorly sized TEVAR: implications and lessons learned
}

\author{
Alex R. Dalal ${ }^{1}$, Shernaz S. Dossabhoy ${ }^{1,2}$, A. Claire Watkins ${ }^{1}$ \\ ${ }^{1}$ Department of Cardiothoracic Surgery, Stanford University School of Medicine, Stanford, CA, USA; ${ }^{2}$ Division of Vascular Surgery, Stanford \\ University School of Medicine, Stanford, CA, USA \\ Correspondence to: A. Claire Watkins, MD, MS. Department of Cardiothoracic Surgery, Stanford University School of Medicine, 300 Pasteur Drive, \\ Falk Building, Mail Code 5407, Stanford, CA 94305, USA. Email: acwat@stanford.edu.
}

Submitted May 18, 2021. Accepted for publication Oct 29, 2021.

doi: 10.21037/acs-2021-taes-87

View this article at: https://dx.doi.org/10.21037/acs-2021-taes-87

\section{Clinical vignette}

A 68-year-old male with history of hypertension, asthma and obstructive sleep apnea presented with sharp chest pain. Medications on admission included four antihypertensives (metoprolol, losartan, nifedipine, and spironolactone) and prednisone. Computed tomography angiogram (CTA) demonstrated uncomplicated type B intramural hematoma (IMH) from the left subclavian artery (LSCA) to the abdominal aorta. After treatment with intravenous then oral antihypertensives, his pain subsided. Repeat imaging on hospital day 6 was stable, and he was discharged. Eighteen days following his original dissection, the patient re-presented with unrelenting, sharp chest and back pain, despite systolic blood pressure of $130 \mathrm{mmHg}$ and pulse rate of 70 beats per minute. CTA revealed evolution of type B $\mathrm{IMH}$ with focal dissection in the distal descending thoracic aorta (DTA). He underwent thoracic endovascular aortic repair (TEVAR) with stent-graft coverage from $2 \mathrm{~cm}$ distal to the LSCA to the celiac artery without complication. His recovery and pre-discharge CTA were unremarkable.

At 6 weeks follow-up, the patient was asymptomatic, however, surveillance CTA demonstrated focal retrograde dissection with aortic dilatation at the proximal edge of the stent-graft distal to the LSCA. He underwent repeat zone 3 TEVAR with a smaller stent-graft. His second procedure and recovery were uneventful. CTA at 2-year demonstrates healed type B dissection with aortic remodeling around his stent-graft.

\section{Aortic stent-graft case planning}

Stent-graft sizing was done using Aquarius iNtuition v4.417 (TeraRecon Inc., Durham, NC, USA). First, a 3-D aortic reconstruction from aortic valve to femoral bifurcation was created to evaluate the aorta's size, shape and course. Manual and automatic blood flow centerlines were compared. Aortic luminal diameter was measured from the inner edge of the aortic wall perpendicularly to the centerline. Manual and automatic measurements were taken at $1 \mathrm{~cm}$ increments, starting at the LSCA. Analysis demonstrated $35.1 \mathrm{~mm}$ aortic diameter at the LSCA, $33.4 \mathrm{~mm}$ at $1 \mathrm{~cm}, 32.1 \mathrm{~mm}$ at $2 \mathrm{~cm}$, and $31.4 \mathrm{~mm}$ at $3 \mathrm{~cm}$ distally, signifying a tapered sealing zone. Goal stentgraft oversizing for this aortic IMH was $5-10 \%$. On-label stent-graft options, based on aortic diameter of $35 \mathrm{~mm}$, included $37 \mathrm{~mm}$ Medtronic Navion or $37 \mathrm{~mm}$ Gore cTAG with Active Control. Other considerations were Terumo RelayPlus or Cook Zenith Alpha. Ultimately, due to its low profile, a $38 \times 34 \times 167 \mathrm{~mm}$ Cook Zenith Alpha was chosen as the distal piece and deployed first and, a $40 \times 167 \mathrm{~mm}$ Cook Zenith Alpha was placed proximally, providing 14-20\% oversized sealing throughout the $2 \mathrm{~cm}$ landing zone. Percutaneous femoral TEVAR proceeded in standard fashion. Intravascular ultrasound (IVUS) was not used. Post-operative CTA demonstrated exclusion of the focal dissections in the distal DTA, reduced IMH thickness and no complications. Notably, the stent-graft was deployed $2.5 \mathrm{~cm}$ distally to the intended landing zone target, the LSCA.

Analysis of the follow-up CTA at 6 weeks demonstrated 35,33 and $32 \mathrm{~mm}$ diameters throughout the proximal DTA. While the original pathology remained treated and the IMH stayed small, the stent-graft had migrated further distally, now positioned $4.5 \mathrm{~mm}$ from the LSCA. Vessel diameter of the proximal aorta, likely injured by the oversized stent-graft, had grown from 38 to $49 \mathrm{~mm}$. 
Reintervention proceeded in the standard fashion. IVUS on a Vision PV 0.035 catheter (Phillips, Netherlands, formerly Volcano, USA) was used to evaluate the quality of the aorta at the LSCA and confirm maximal aortic luminal measurements during systole. Measurements confirmed $35 \mathrm{~mm}$ landing zone at the LSCA with minimal IMH. A $37 \times 150 \mathrm{~mm}$ Gore Active Control cTAG was deployed immediately distal to the LSCA, followed by $40 \times$ $150 \mathrm{~mm}$ Gore cTAG to lock the new graft into the original $40 \mathrm{~mm}$ Zenith Alpha stent-graft. The patient's course was unremarkable, and imaging remains stable at 2-year follow-up.

\section{Comments}

Precise case planning and stent-graft sizing is critical in TEVAR. The stent-graft-induced aortic injury seen here was likely due to oversizing, slight IMH disease, tortuosity in the proximal landing zone, prednisone use and imprecise stent deployment. With oversizing goals for aneurysm being $10-20 \%$ and $5-10 \%$ for dissection, sizing for dissection is more challenging with a narrower therapeutic range. Surgeons occasionally must decide between a stentgraft that is $<5 \%$ or $>10 \%$ oversized, often in emergent settings. Goal oversizing and landing zone length for a given pathology has been defined by clinical studies and is available in each device's instructions for use (IFU). In practice, deciding between two stent-graft sizes requires consideration of several factors, with little information regarding the risk of each choice.

This case did not change our standard protocol but emphasizes the need to strictly follow sizing principles. Oversizing $14-20 \%$ in the first intervention compared to $5-14 \%$ in the second was critical to avoiding aortic injury. Oversizing $5-14 \%$ across the entire $2 \mathrm{~cm}$ landing zone, which often significantly tapers, is also essential to prevent stent migration.

Anatomic considerations in stent sizing include the health, length, and shape of aortic landing zones. The longer and healthier the landing zone, the more likely a $3-6 \%$ oversized stent-graft will be technically successful. The more diseased a landing zone, the higher the risk of injury from oversizing.

Tortuosity in a landing zone leads to more challenging case planning and sizing, as well as less predictable stentgraft positioning and differing radial force on the vessel intima. While the degree of oversizing is an important consideration to avoid retrograde type A dissection (RTAD), an arch landing zone or ascending aortic diameter $>4.0 \mathrm{~cm}$ is a stronger predictor (1). The clinical implications of potential endoleak change greatly in the setting of a rupture, lending to the more oversized stent-graft choice. Significant, unstable rupture may be the only clinical setting in which endoleak is more harmful than RTAD. In traumatic aortic transection or aortic rupture, hypovolemia and vasoconstriction will reduce aortic diameters and together with under-resuscitation can lead to stent-graft undersizing (2).

Measurement methodology is also important for properly selecting stent-graft size. Sizing relies on highquality CT imaging, preferably with slice thickness $\leq 1 \mathrm{~mm}$. Additionally, ECG-gating is useful as aortic diameters can change up to $20 \%$ throughout the cardiac cycle. This case did not initially utilize IVUS; a tool shown to improve TEVAR outcomes (3). While CTA and IVUS measurements often differ, with the latter tending toward larger diameters (4), IVUS offers a real-time assessment of the aorta that can alert surgeons to progression of pathology or interval aortic dilation, assisting in surgical decisionmaking during TEVAR.

In conclusion, careful consideration of clinical and anatomic features as well as multiple modalities of measurement, contributes to safe and accurate stent-graft sizing for TEVAR.

\section{Acknowledgments}

Funding: None.

\section{Footnote}

Conflicts of Interest: The authors have no conflicts of interest to declare.

Open Access Statement: This is an Open Access article distributed in accordance with the Creative Commons Attribution-NonCommercial-NoDerivs 4.0 International License (CC BY-NC-ND 4.0), which permits the noncommercial replication and distribution of the article with the strict proviso that no changes or edits are made and the original work is properly cited (including links to both the formal publication through the relevant DOI and the license). See: https://creativecommons.org/licenses/by-nc-nd/4.0/.

\section{References}

1. Yammine H, Briggs CS, Stanley GA, et al. Retrograde type 
A dissection after thoracic endovascular aortic repair for type B aortic dissection. J Vasc Surg 2019;69:24-33.

2. Lortz J, Tsagakis K, Rammos C, et al. Hemodynamic changes lead to alterations in aortic diameters and may challenge further stent graft sizing in acute aortic syndrome. J Thorac Dis 2018;10:3482-9.

3. Lortz J, Tsagakis K, Rammos C, et al. Intravascular

Cite this article as: Dalal AR, Dossabhoy SS, Watkins AC. Poorly sized TEVAR: implications and lessons learned. Ann Cardiothorac Surg 2021;10(6):804-806. doi: 10.21037/acs-2021taes-87 ultrasound assisted sizing in thoracic endovascular aortic repair improves aortic remodeling in Type B aortic dissection. PLoS One 2018;13:e0196180.

4. Han SM, Elsayed RS, Ham SW, et al. Comparison of intravascular ultrasound- and centerline computed tomography-determined aortic diameters during thoracic endovascular aortic repair. J Vasc Surg 2017;66:1184-91. 